\title{
Laser Guide Star Based Astrophysics at Lick Observatory
}

C.E. Max, D.T. Gavel, H.W. Friedman, S.S. Olivier, B.A. Macintosh, J.M. Brase, K. Avicola, S. Gibbard and J. An 


\section{DISCLAIMER}

This document was prepared as an account of work sponsored by an agency of the United States Government. Neither the United States Government nor the University of California nor any of their employees, makes any warranty, express or implied, or assumes any legal liability or responsibility for the accuracy, completeness, or usefulness of any information, apparatus, product, or process disclosed, or represents that its use would not infringe privately owned rights. Reference herein to any specific commercial product, process, or service by trade name, trademark, manufacturer, or otherwise, does not necessarily constitute or imply its endorsement, recommendation, or favoring by the United States Government or the University of California. The views and opinions of authors expressed herein do not necessarily state or reflect those of the United States Government or the University of California, and shall not be used for advertising or product endorsement purposes.

Work performed under the auspices of the U. S. Department of Energy by the University of California Lawrence Livermore National Laboratory under Contract W-7405-Eng-48.

This report has been reproduced directly from the best available copy.

Available to DOE and DOE contractors from the

Office of Scientific and Technical Information

P.O. Box 62, Oak Ridge, TN 37831

Prices available from (423) 576-8401

http://apollo.osti.gov/bridge/

Available to the public from the National Technical Information Service

U.S. Department of Commerce 5285 Port Royal Rd., Springfield, VA 22161 http://www.ntis.gov/

OR

Lawrence Livermore National Laboratory Technical Information Department's Digital Library http://www.llnl.gov/tid/Library.html 


\title{
Laser Guide Star Based Astrophysics at Lick Observatory
}

\author{
3-year Final Report \\ Exploratory Research in the Institutes (ERI) Program \\ 97-ERD-037 \\ Institute for Geophysics and Planetary Physics
}

Claire E. Max (Principal Investigator)

Donald T. Gavel, Herbert W. Friedman, Scot S. Olivier, Bruce

A. Macintosh, James M. Brase, Kenneth Avicola, Seran Gibbard, and Jong An (Co-investigators)

\begin{abstract}
The resolution of ground-based telescopes is typically limited to $\sim 1$ second of arc because of the blurring effects of atmospheric turbulence. Adaptive optics (AO) technology senses and corrects for the optical distortions due to turbulence hundreds of times per second using high-speed sensors, computers, deformable mirror, and laser technology. The goal of this project is to make AO systems widely useful astronomical tools providing resolutions up to an order of magnitude better than current, ground-based telescopes. Astronomers at the University of California Lick Observatory at Mt. Hamilton now routinely use the LLNL developed AO system for high resolution imaging of astrophysical objects. We report here on the instrument development progress and on the science observations made with this system during this 3-year ERI project.
\end{abstract}

\section{Introduction}

The uncorrected resolution of a ground-based telescope is typically limited to about one second of arc on the sky because of the blurring effects of atmospheric turbulence. In terms of the theoretical diffraction-limit, this is no better resolution than a telescope with a $10 \mathrm{~cm}$ diameter aperture. Adaptive optics (AO) technology senses and corrects for the optical distortions hundreds of times per second using high-speed sensors, computers, and deformable mirror and laser technology originally developed for the atomic vapor laser isotope separation (AVLIS) program. With present day AO technology, telescopes can reach diffraction-limited resolution, $\theta \cong \lambda / \mathrm{d}$, at near infrared wavelengths, 1-2.5 microns. This is wavelength band is of significant interest to astrophysicists studying the physics of galaxy formation in the early universe, star and solar system formation, nearby planetary systems (outside our own solar system), the atmospheres of gas giant planets, and many other astrophysical phenomena. Our system at the University of California 
Lick Observatory three-meter Shane Telescope* improves resolution to 0.1 arcsecond, a factor of ten over uncorrected seeing.

The goal of this project is to make $\mathrm{AO}$ systems widely useful astronomical tools. In FY 1998 and 1999, the AO system on the 3-meter telescope was significantly upgraded to improve the quality and efficiency of science observations ${ }^{1,2,3}$. The AO system contains a laser, which can produce an artificial beacon for sensing and correcting the atmospheric turbulence in regions of the sky where natural guide stars are not available. In engineering tests in 1996, the AO system's laser guide star was world's first to demonstrate corrected images ${ }^{4}$. We've also shown excellent results using natural guide stars as dim as $12^{\prime}$ th magnitude in engineering tests and science observations since 1996. Today, laser guide star systems have been installed and are being planned on a number of other telescopes around the world, including the Keck 10-meter telescope on Mauna Kea in Hawaii ${ }^{5}$.

During 1998 and 1999, the AO system was used in dedicated science observation runs by a number of University of California faculty and graduate student observers. Astronomy projects included imaging of newly-forming stars, a search for dim binary star companions, measurements of the mass density of nearby galaxies, characterizing gasgiant atmospheres, and imaging galaxies containing active nuclei.

\section{Instrumental Improvements and Technology Development}

The Lick AO system underwent improvements in 1998 and 1999 to better accommodate routine science observations. In 1998 we incorporated a new infrared science camera, developed over the previous two years in collaboration with Professor James Graham of the University of California, Berkeley, which is optimized for the higher resolution capability provided by the $\mathrm{AO}$ system $^{6}$. During a break in observing in the winter of 1998, we brought the AO system back to LLNL and modified the AO bench optical layout to improve IR observing ${ }^{2}$. The new layout allows wide field observations where the natural guide star (usually a bright nearby star in our galaxy) is separated from the science object of interest (for example, an extragalactic object such as a quasar). Automated steering mirrors were incorporated to allow for "nodding" of the telescope over the science field, which is a common observing technique that helps eliminate IR sensor pattern noise, but would otherwise complicate locking the AO control loop on a fixed natural guide star. The software interface and optical bench automation were also upgraded at that time so that the system can be set up, calibrated, and operated by one person from a computer workstation. Over the course of this project, we have developed and tested novel calibration procedures to reduce the internal $\mathrm{AO}$ system aberrations to accommodate the demand of diffraction-limited image quality ${ }^{7,8,9}$.

Most extragalactic astronomy and even a large fraction of the study of galactic objects such as young stellar regions, nebular remains of supernovea, etc. will benefit greatly by use of the laser guide star because most targets are not near 12'th magnitude or brighter natural guide stars. Our 20 Watt dye laser, mounted on the side of the Shane telescope, produces the equivalent of a 9'th magnitude artificial guide star at any direction the

\footnotetext{
${ }^{*}$ Lick Observatory is located on top of Mt. Hamilton, near San Jose, California.
} 
telescope points. The dye laser is tuned to the $589 \mathrm{~nm}$ resonant line of atomic sodium so that it causes the sodium in the earth's mesospheric layer at $90 \mathrm{~km}$ altitude to fluoresce.

In our efforts to make the laser guidestar system a routinely usable instrument, we improved and tested a number of laser subsystem components during this program. Procedures for aligning the pump lasers were improved, making them more stable in power output. Temperature control was installed in the laser room housing the master oscillator, which improved its wavelength stability. Improved diagnostic systems in the laser beam line helped bring the laser launch telescope into better alignment ${ }^{10,11}$.

\section{Science Programs}

Astronomers Andrea Ghez (UCLA), James Graham (UCB), and their graduate students Jenny Patience (UCLA) and Mike Liu (UCB), studied the formation rate of binary stars in the Pliedes and Hyades clusters and other star-forming regions of our galaxy $^{12}$. There is some evidence that the percentage of young stars in binary systems is higher than the percentage of mature stars in binary systems. This would imply that forming star systems are dynamically unstable and eventually kick out their orbiting companions. One young star system, LkHa 234 shown in Figure 1, has as many as 5 coorbiting stars ${ }^{13}$. This image demonstrates the resolving power of adaptive optics. Previous to these observations, ground-based observations resolved only two stars in LkHa 234.

Bruce Macintosh (LLNL) and Mike Liu (UCB) have been searching for low mass companion stars called brown dwarves. These are stars just above the mass limit to initiate nuclear fusion and are quite difficult to observe because of their low luminosity. They are scientifically interesting because they may be an important component of the mass of galaxies and their population densities can lead to a better understanding of the formation and evolution of galaxies. The AO system's resolving power acts to concentrate the dim star's light spatially, which helps to overcome background noise thus extending the lower limit of detectable luminosity.

Mike Liu has also been observing dim stellar companions to stars which are known from previous observations to have planets ${ }^{12,14}$. There is a question of whether it is common for dynamically stable planetary systems to form around binary stars, or if planets are to be found only around single stars. This has profound implications on the statistics of planetary systems, since most stars are doubles. A spin-off technological benefit of this work is in the development of techniques that will be used eventually for detecting the planets themselves against the glare of their parent star. Detecting planets will require much larger telescopes and $\mathrm{AO}$ systems, however the development of the internal calibration technology and understanding of the point spread functions of adaptive optics, driven by the need to observe faint objects next to bright ones, are crucial to future success in this area.

Imke de Pater (UCB) and graduate student Henry Roe have been studying the atmospheric chemistry of Neptune ${ }^{15}$. The AO system can resolve the dark regions of the atmosphere separate from the bright high-altitude clouds, allowing narrow band filters to probe to particular altitudes of the atmosphere in the unclouded regions (Figure 2). Understanding the optical depth profiles, and altitudes of various haze layers, allows us 
to gain better understanding of the energy balance between solar flux and internal heat sources on Neptune.

Extragalactic astronomy is perhaps the most challenging of adaptive optics observations because these objects, galaxies and quasars, are not themselves bright enough to serve as natural guide stars. This year, the approach has been to select extragalactic targets that are fortuitously near (as they appear to us) bright stars in our own galaxy. Andreas Quirrenbach (UCSD) has been observing the centers of several types of galaxies. Radio galaxies emit brightly in the radio wavelengths because the host galaxies have an energetic core. AO infrared observations at high resolution allow the host galaxy to be identified and aligned to the radio observations. Some galaxies have quasars at their nucleus. A quasar is a very bright object associated only with very high red shift (young with respect to the age of the universe) galaxies. The AO system helps resolve the structure of the host galaxy giving insight into galaxy formation physics in the early universe.

While the laser has generated excellent $\mathrm{AO}$ correction during engineering tests, it has been used only a few science observations. The companion to the 15'th magnitude star FS Tau, at 0.1 arcsecond separation, was imaged using laser guided AO during November 1998 (Figure 3). This is one of the many T-Tauri type young stellar binaries. The observation demonstrates the capability of adaptive optics to probe to close binary separations. Completion of this science project will require a statistical survey of such objects, which is work in progress.

\section{Publications}

Attached are to this report is a series of journal publications documenting Lick adaptive optics system instrument development during this LDRD project.

A number of astronomers have reported on observations using the Lick adaptive optics system. These are references ${ }^{12-15}$.

\section{References}

${ }^{1}$ Olivier, S. S., Gavel, D. T., Friedman, H.W., Max, C.E., An, J.R., Avicola, K., Bauman, B. J., Brase, J. M., Campbell, E.W., Carrano, C.J., Cooke, J. B., Freeze, G.J., Gates, E. L., Kanz, V.K., Kuklo, T.C., Macintosh, B. A., Newman, M.J., Pierce, E.L., Waltjen, K.E., and Watson, J.A., Improved performance of the laser guide star adaptive optics system at Lick Observatory, UCRL-JC-134956, Proc. of the SPIE, Vol. 3762, 2-7, 1999.

2 Bauman, B.J., Gavel, D.T., Waltjen, K.E., Freeze, G.J., Keahi, K.A., Kuklo, T.C., Lopes, S.K., Newman, M.J., and Olivier, S.S., UCRL-JC-134970, New optical design of adaptive optics system at Lick Observatory, Proc. of the SPIE, Vol. 3762, 194200, 1999.

3 Gavel, D. T. Friedman, H. W., and Bauman, B.J., Lick sodium laser guide star: performance during the 1998 LGS observing campaign, UCRL-JC-135002, Proc. of the SPIE, Vol. 3762, 20-27, 1999.

${ }^{4}$ Max, C. E., Olivier, S. S., Friedman, H. W., An, J., Avicola, K., Beeman, B. V., Bissinger, H. D., Brase, J. M., Erbert, G. V., Gavel, D. T., Kanx, V. K., Liu, M. C., 
Macintosh, B. A., Neeb, K. P., Patience, J., Waltjen, K. E., Image improvement from a sodium-layer laser guide star adaptive optics system, UCRL-ID-126000, Science, 277, 1649-1652, 1997.

${ }^{5}$ Wizinowich, P. L., Acton, D. S., Gregory, T., Stomski, P. J., An, J. R., Avicola, K., Brase, J. M., Friedman, H. W., Gavel, D. T., Max, C. E., Status of the W.M. Keck Adaptive Optics Facility, Proc. of the SPIE, Vol. 3353, 568-578, 1998.

${ }^{6}$ Lloyd, J. P., Liu, M. C., Macintosh, B. A., Deich, W. T., Severson, S. A., Graham, J. R., IRCAL: the infrared camera for adaptive optics at Lick Observatory, to be presented at SPIE 4008, March 2000.

${ }^{7}$ Campbell, E. W., Bauman, B.J., Swieder, D.R., and Olivier, S.S., High-accuracy calibration of an adaptive optics system using a phase-shifting diffraction interferometer, UCRL-JC-134805, Proc. of the SPIE, Vol. 3762, 237-244, 1999.

${ }^{8}$ Gavel, D. T., Bauman, B.J., Campbell, E.W., Carrano, C.J., and Olivier S.S., Practical comparison of phase diversity to interferometry in measuring the aberrations in an adaptive optics system, UCRL-JC-135003, Proc. of the SPIE, Vol. 3762, 266-268, 1999.

${ }^{9}$ Carrano, C. J., Olivier, S. S., Brase, J. M., Macintosh, B. A., An, J. R., Phase retrieval techniques for adaptive optics, UCRL-JC-130921, Proc. SPIE, Vol. 3353, 658-667, 1998.

${ }^{10}$ Gavel, D. T. Friedman, H. W., and Bauman, B.J., Lick sodium laser guide star: performance during the 1998 LGS observing campaign, UCRL-JC-135002, Proc. of the SPIE, Vol. 3762, 20-27, 1999.

${ }^{11}$ Gavel, D. T., and Friedman, H. W., Measurements of the Lick Observatory sodium laser guide star, UCRL-JC-130922, Proc. SPIE Vol. 3353, 254-259, 1998.

${ }^{12}$ Patience, J., Ghez, A. M., White, R. J., McCabe, C., Macintosh, B., Liu, M. C., Graham, J. R., Max, C. E., Gavel, D., Olivier, S., Rudy, R., Puetter, R., Matthews, K., and Weinberger, A. J., A High-Resolution Search for Stellar Companions to Stars with Planets, American Astronomical Society Meeting 193, 97.08, 1998.

${ }^{13}$ Liu, M. C., and Graham, J. R., Multiplicity Revealed: Adaptive Optics Imaging of the Young Outflow Source LkHa 234, submitted to The Astrophysical Journal, 2000.

${ }^{14}$ Marcy , G. W., Butler, R. P., Vogt, S. S., Fischer, D., and Liu, M. C., Two new candidate planets in eccentric orbits, The Astrophysical Journal, v. 520, 239-247, 1999.

${ }^{15}$ Roe, H., Gavel, D., Max, C., de Pater, I., Gibbard, S., and Macintosh, B., Observations of Neptune's Tropospheric Cloud Layer with the Lick Adaptive Optics System, submitted to Astrophysical Journal, 1999. 


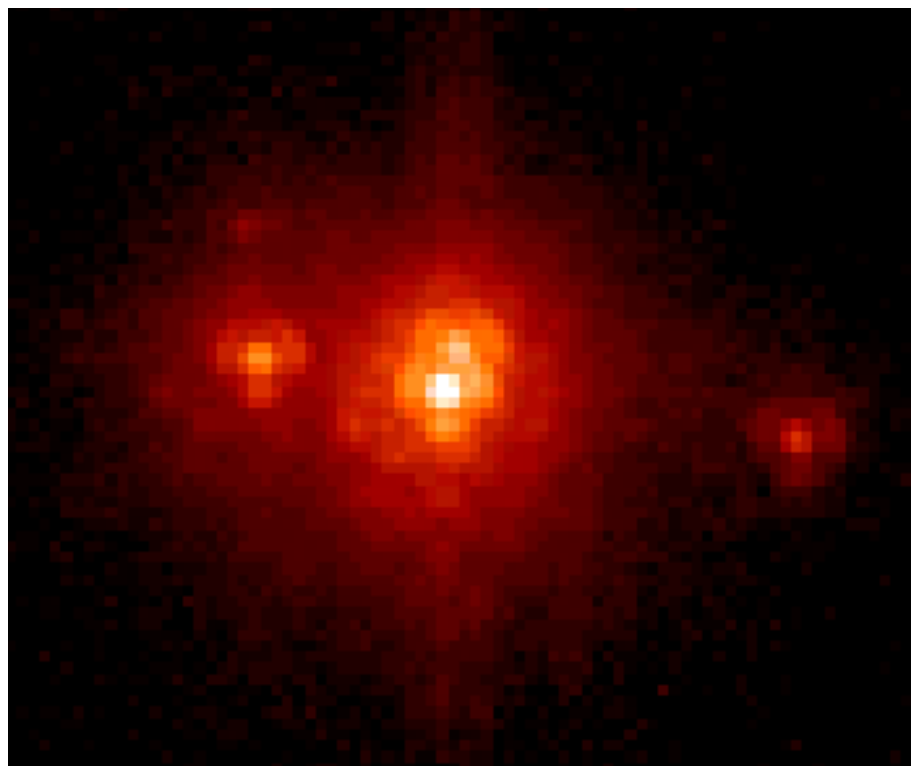

Figure 1. Adaptive optics image of $\mathrm{LkH} \alpha$ 234, showing five stars in a young stellar system. Previous non-AO observations identified this object only as a binary. Such mult-star systems are usually dynamically unstable, so eventually several of these stars will be ejected from the system. The "ring" around each star is a characteristic of diffraction-limited imaging (the Airy ring). The bright "halo" is residual scattered light due to incomplete AO correction of the atmospheric turbulence. From Liu, et. al., 2000. ${ }^{13}$ 


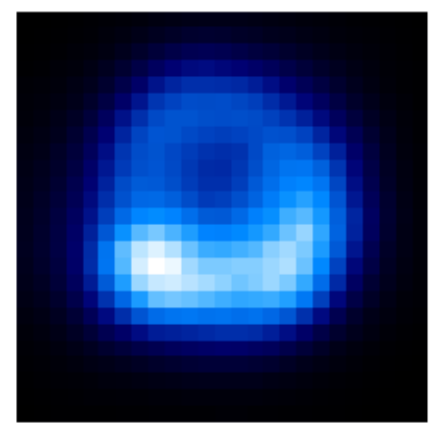

Fig. 1a: J-tiller June 1997

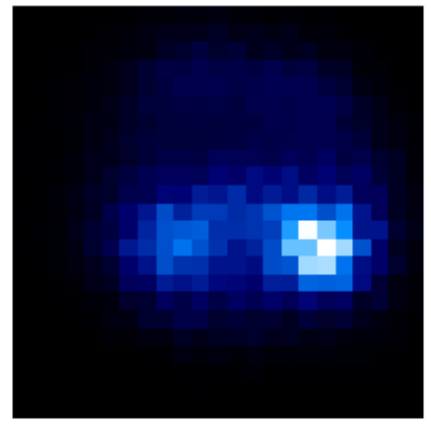

Fig. 1e: J-tiller July 1998

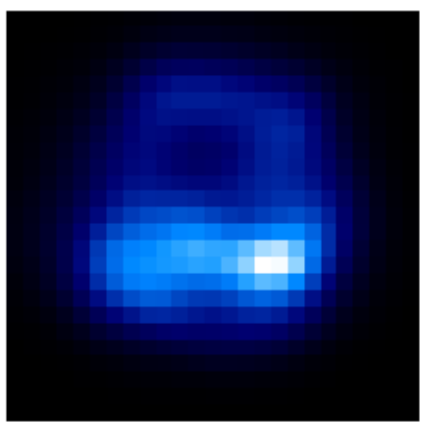

Fig. 1b: H-tilker June 1997

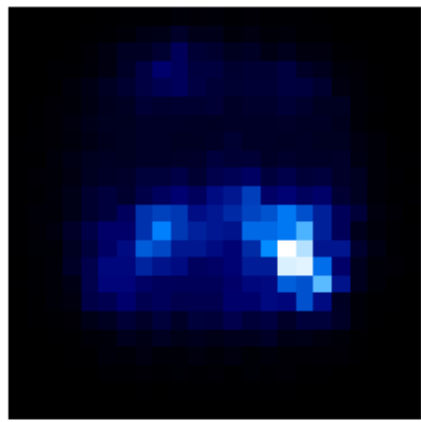

Fig. 1d: H-tilier July 1998

Figure 2. Images of Neptune taken with the Lick adaptive optics system, showing variability of storms in the upper atmosphere. The during the present Neptunian season, southern hemisphere summer, storms are prevalent at the southern latitudes, streaming out along latitude lines. Polar hazes are also evident. From Roe, et. al., 1999. ${ }^{15}$ 

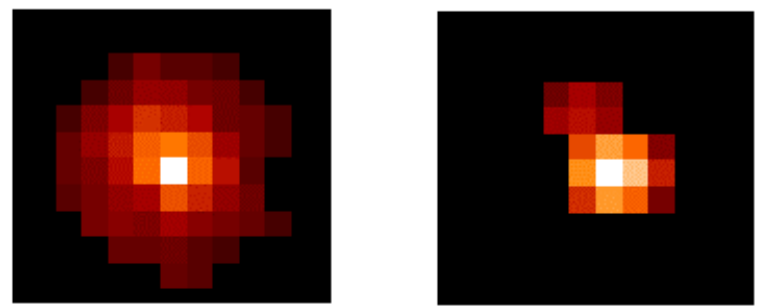

Figure 3. A binary companion to a young T-Tauri type star, FS Tau, is detected with the adaptive optics system in laser guide star mode. FS Tau is a $15^{\prime}$ th magnitude star, too $\operatorname{dim}$ to use as a natural guide star. The companion, separated by about 0.13 arcsecond, is about a factor of 10 dimmer. The image at the left was taken in $\mathrm{H}$ band $(\lambda=1.6 \mu \mathrm{m})$ with the Lick facility infrared camera, LIRC-II, in November 1998. The image at the right is the same image but deconvolved using a known point-source single star, also corrected with laser guide star adaptive optics, as the point spread function reference. 


\section{Papers attached to this report}

Olivier, S. S., Gavel, D. T., Friedman, H.W., Max, C.E., An, J.R., Avicola, K., Bauman, B. J., Brase, J. M., Campbell, E.W., Carrano, C.J., Cooke, J. B., Freeze, G.J., Gates, E. L., Kanz, V.K., Kuklo, T.C., Macintosh, B. A., Newman, M.J., Pierce, E.L., Waltjen, K.E., and Watson, J.A., Improved performance of the laser guide star adaptive optics system at Lick Observatory, UCRL-JC-134956, Proc. of the SPIE, Vol. 3762, 2-7, 1999.

Gavel, D. T. Friedman, H. W., and Bauman, B.J., Lick sodium laser guide star: performance during the 1998 LGS observing campaign, UCRL-JC-135002, Proc. of the SPIE, Vol. 3762, 20-27, 1999.

Bauman, B.J., Gavel, D.T., Waltjen, K.E., Freeze, G.J., Keahi, K.A., Kuklo, T.C., Lopes, S.K., Newman, M.J., and Olivier, S.S., New optical design of adaptive optics system at Lick Observatory, UCRL-JC-134970, Proc. of the SPIE, Vol. 3762, 194-200, 1999.

Campbell, E. W., Bauman, B.J., Swieder, D.R., and Olivier, S.S., High-accuracy calibration of an adaptive optics system using a phase-shifting diffraction interferometer, UCRL-JC-134805, Proc. of the SPIE, Vol. 3762, 237-244, 1999.

Gavel, D. T., Bauman, B.J., Campbell, E.W., Carrano, C.J., and Olivier S.S., Practical comparison of phase diversity to interferometry in measuring the aberrations in an adaptive optics system, UCRL-JC-135003, Proc. of the SPIE, Vol. 3762, 266-268, 1999.

Max, C. E., Macintosh, B. A., Olivier, S. S., Gavel, D. T., and Friedman, H. W., Observing techniques for astronomical laser guide star adaptive optics, UCRL-JC130702, Proc. SPIE Vol. 3353, 277-281, 1998.

Gavel, D. T., and Friedman, H. W., Measurements of the Lick Observatory sodium laser guide star, UCRL-JC-130922, Proc. SPIE Vol. 3353, 254-259, 1998.

Carrano, C. J., Olivier, S. S., Brase, J. M., Macintosh, B. A., An, J. R., Phase retrieval techniques for adaptive optics, UCRL-JC-130921, Proc. SPIE, Vol. 3353, 658-667, 1998.

Max, C. E., Olivier, S. S., Friedman, H. W., An, J., Avicola, K., Beeman, B. V., Bissinger, H. D., Brase, J. M., Erbert, G. V., Gavel, D. T., Kanx, V. K., Liu, M. C., Macintosh, B. A., Neeb, K. P., Patience, J., Waltjen, K. E., Image improvement from a sodium-layer laser guide star adaptive optics system, UCRL-ID-126000, Science, 277, 1649-1652, 1997. 